\title{
Analysis of Moment Transmitted by Universal Joint in Varying Operating Condition
}

\author{
Changlin Feng, Deshi Wang and Yongyong Zhu \\ Department of Weaponry Engineering, Naval University of Engineering, wuhan 430033, china \\ fcl_325@126.com
}

Keywords: transmitted moment, universal joint, varying operating condition, vector projection, fluctuation

\begin{abstract}
The moments transmitted by a universal joint in varying operating condition was investigated. A model of shaft system driven by a universal joint which consists of a driving shaft, a driven shaft and a cross piece is chose. Both structure misalignment and error misalignment between the two shafts at the universal joint was considered, and the study was based on the assumption that friction, inertia and elastic forces of the cross piece were disregarded. Two pairs of Euler angles were introduced to denote structure misalignment and error misalignment respectively, sets of reference frames were built up on the driving shaft, driven shaft and cross piece. Using direction cosine matrixes to describe the relation between different frames, then the expressions of components of the transmitted moment acting on the driven shaft were obtained via method of vector projection. Based on engineering fact, the transmitted moment model was simplified with respect to different misalignment conditions, such as only structure misalignment or only error misalignment is considered. Then for different misalignment situations, the lateral and torsional components of the transmitted moment acting on the driven shaft were simulated through numerical examples. It turns out that both the lateral moment and torque components will fluctuate based on the import torque, and that due to accelerating of the driving shaft, the fluctuation of transmitted moment will be more distinct, consequently, more complicated nonlinear dynamics behavior will arise.
\end{abstract}

\section{Introduction}

Misalignment in rotating machinery is the second most common malfunction after unbalance, so the problem of misalignment as encountered in rotating machinery is of great concern to designers and maintenance engineers [1]. There are three kinds of misalignment, the parallel misalignment, the angular misalignment and coupled parallel- angular misalignment. Angular misalignment is a condition in which the driving machine shaft and the driven machine shaft have different angular orientations. Universal joint, which can transmit moment and energy from a driving shaft to a driven shaft, is commonly used as a source of angular misalignment. In rotor system driven by universal joint, the fluctuating angular velocity ratio and impact characteristic of transmission moment due to the joint can cause nonlinear vibration and dynamic stabilities issue, especially, during start-up process of the system, these issues will appear more obviously. Studying moment transmission by a universal joint during start-up process is significative for confirming nonlinear dynamic behavior of system.

There are some works on moment transmission by a universal joint. I. Porat [2] developed a general theory, which based on the assumption of a static system with friction, inertia and elastic forces disregarded, for calculation of the moments transmitted by an arbitrary universal joint. It turns out that a moment component normal to the shaft is unavoidable. T. Iwatsubo [3], A. J. Mazzei [4] and $\mathrm{H}$. Ota [5] also investigated the moments transmitted by a universal joint before they modeling the motion equation of shaft driven by a universal joint. M. Kato [6] and P. P. Sheu [7] studied the transmitted moments in the shaft system driven by a universal joint with friction regarded and in the shaft system driven by two universal joints respectively. 
In the present paper, the moments (the normal component as well as those along the rotation axes) transmitted by a universal joint in varying operating condition was investigated. Both structure misalignment and error misalignment between the two shafts at the universal joint was considered, the expressions of components of the transmitted moment acting on the driven shaft were obtained via method of vector projection, at last, the model was simulated through numerical examples.

\section{Frames of Reference and Angularities}

For the sake of completeness a discussion the moment transmission by a universal joint, the device consists of a driving shaft, a driven shaft and a cross piece is given. A pair of Euler angles $\varphi, \delta$ are introduced to denote structure misalignment and another pair of Euler angles $\beta, \gamma$ are introduced to denote error misalignment. Sets of reference frames were built up on the driving shaft, driven shaft and cross piece as shown in Fig. 1. The description of these frames and the relation between these frames, consist of direction cosine matrixes $C_{\varphi}, C_{\delta}, C_{\beta}, C_{\gamma}$, can be seen in references [4].

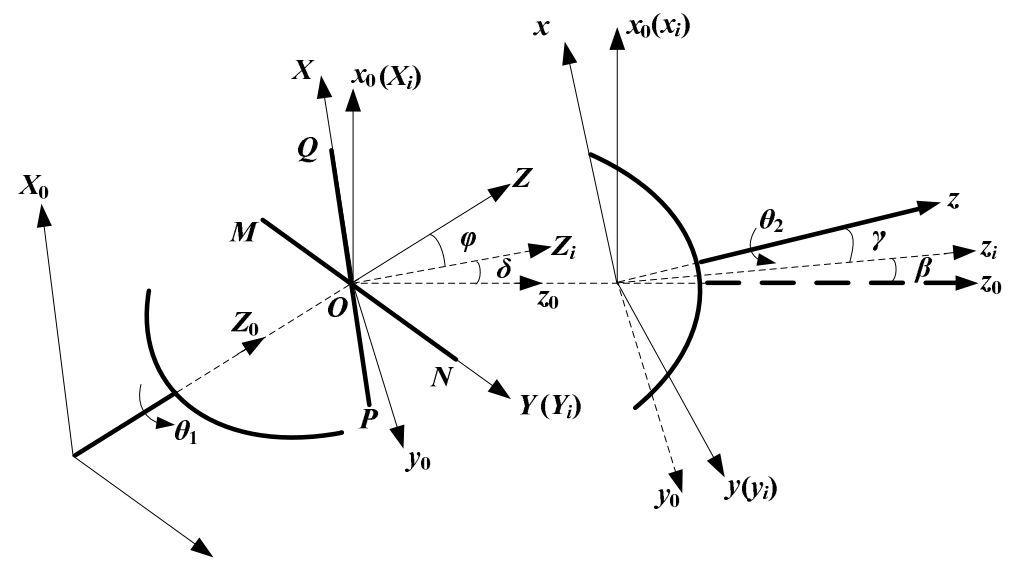

Fig. 1. Reference frames

\section{Moments Transmission}

A torque $\vec{T}_{0}=T_{0} \hat{K}$ is transmitted along the driving shaft, the angular acceleration of the driving shaft is $\alpha$, when the driving shaft rotating $\theta_{1}$, the driven shaft will rotate $\theta_{2}$. The unit vector $\hat{e}_{1}$ is directed along $\overrightarrow{O N}$, the unit vector $\hat{e}_{2}$ is directed along $\overrightarrow{O Q}$, the unit vector $\hat{e}_{3}$ is directed along the actual driven shaft, and the normal unit vector to the cross piece is $\hat{e}_{n}$. $\hat{e}_{1}$ can be obtained through a rotation of $\theta_{1}$, in the $x y z$ frame, it is given by

$$
\begin{aligned}
& \hat{e}_{1(x y z)}=\left(\begin{array}{l}
e_{1 x} \\
e_{1 y} \\
e_{1 z}
\end{array}\right)=\boldsymbol{C}_{\gamma} \boldsymbol{C}_{\beta} \boldsymbol{C}_{\delta} \boldsymbol{C}_{\varphi}\left(\begin{array}{c}
-\sin \theta_{1} \\
\cos \theta_{1} \\
0
\end{array}\right) \\
& =\left(\begin{array}{c}
-\sin \theta_{1}(\cos \gamma \cos \delta+\sin \gamma \cos \beta \sin \delta)+\cos \theta_{1}[\sin \gamma \sin \beta \cos \varphi+(\cos \gamma \sin \delta-\sin \gamma \cos \beta \cos \delta) \sin \varphi] \\
\sin \theta_{1} \sin \beta \sin \delta+\cos \theta_{1}(\cos \beta \cos \varphi+\sin \beta \cos \delta \sin \varphi) \\
-\sin \theta_{1}(\sin \gamma \cos \delta-\cos \gamma \cos \beta \sin \delta)-\cos \theta_{1}[\cos \gamma \sin \beta \cos \varphi-(\sin \gamma \sin \delta+\cos \gamma \cos \beta \cos \delta) \sin \varphi]
\end{array}\right)
\end{aligned}
$$

The components of $\hat{e}_{3}$ in the $x y z$ frame are given by

$$
\hat{e}_{3(x y z)}=\left(\begin{array}{lll}
0 & 0 & 1
\end{array}\right)^{T}
$$


From geometric constrains on the structure of universal joint, $\hat{e}_{2}$ is perpendicular to $\hat{e}_{1}$ and $\hat{e}_{3}$, but $\hat{e}_{1}$ is not perpendicular to $\hat{e}_{3}$. So $\hat{e}_{2}$ can be obtained via cross product between $\hat{e}_{1}$ and $\hat{e}_{3}$ as

$$
\hat{e}_{2(x y z)}=\left(\begin{array}{c}
e_{2 x} \\
e_{2 y} \\
e_{2 z}
\end{array}\right)=\frac{\hat{e}_{3(x y z)} \times \hat{e}_{1(x y z)}}{\left|\hat{e}_{3(x y z)} \times \hat{e}_{1(x y z)}\right|}=\frac{1}{\sqrt{e_{1 x}^{2}+e_{1 y}^{2}}}\left(\begin{array}{c}
-e_{1 y} \\
e_{1 x} \\
0
\end{array}\right)
$$

$\hat{e}_{n}$ is perpendicular to $\hat{e}_{1}$ and $\hat{e}_{2}$, and $\hat{e}_{1}$ is also perpendicular to $\hat{e}_{2}$. So $\hat{e}_{n}$ can be obtained via cross product between $\hat{e}_{1}$ and $\hat{e}_{2}$ as

$$
\hat{e}_{n(x y z)}=\left(\begin{array}{l}
e_{n x} \\
e_{n y} \\
e_{n z}
\end{array}\right)=\hat{e}_{1(x y z)} \times \hat{e}_{2(x y z)}=\frac{1}{\sqrt{e_{1 x}^{2}+e_{1 y}^{2}}}\left(\begin{array}{c}
-e_{1 x} e_{1 z} \\
-e_{1 y} e_{1 z} \\
e_{1 x}^{2}+e_{1 y}^{2}
\end{array}\right)
$$

The components of $\hat{e}_{n}$ in the $x y z$ frame are given by

$$
\begin{aligned}
& e_{n x}=\frac{1}{\xi}\left\{\begin{array}{l}
-\sin \theta_{1}(\cos \gamma \cos \delta+\sin \gamma \cos \beta \sin \delta) \\
+\cos \theta_{1}[\sin \gamma \sin \beta \cos \varphi+(\cos \gamma \sin \delta-\sin \gamma \cos \beta \cos \delta) \sin \varphi]
\end{array}\right\} \\
& \left\{\begin{array}{l}
\sin \theta_{1}(\sin \gamma \cos \delta-\cos \gamma \cos \beta \sin \delta) \\
+\cos \theta_{1}[\cos \gamma \sin \beta \cos \varphi-(\sin \gamma \sin \delta+\cos \gamma \cos \beta \cos \delta) \sin \varphi]
\end{array}\right\} \\
& e_{n y}=\frac{1}{\xi}\left\{\sin \theta_{1} \sin \beta \sin \delta+\cos \theta_{1}(\cos \beta \cos \varphi+\sin \beta \cos \delta \sin \varphi)\right\} \\
& \left\{\begin{array}{l}
\sin \theta_{1}(\sin \gamma \cos \delta-\cos \gamma \cos \beta \sin \delta) \\
+\cos \theta_{1}[\cos \gamma \sin \beta \cos \varphi-(\sin \gamma \sin \delta+\cos \gamma \cos \beta \cos \delta) \sin \varphi]
\end{array}\right\} \\
& e_{n z}=\xi \sqrt{\left.\begin{array}{l}
\left\{\sin \theta_{1} \sin \beta \sin \delta+\cos \theta_{1}(\cos \beta \cos \varphi+\sin \beta \cos \delta \sin \varphi)\right\}^{2} \\
+\left\{\begin{array}{l}
-\sin \theta_{1}(\cos \gamma \cos \delta+\sin \gamma \cos \beta \sin \delta) \\
+\cos \theta_{1}[\sin \gamma \sin \beta \cos \varphi+(\cos \gamma \sin \delta-\sin \gamma \cos \beta \cos \delta) \sin \varphi]
\end{array}\right.
\end{array}\right\}^{2}}
\end{aligned}
$$

The moment of inertia of the driving shaft is $J_{1}$, one can write an equation for the static moment balance about $X$ as

$$
T_{0}-M \hat{e}_{n} \cdot \hat{K}=J_{1} \alpha
$$

Here, $\hat{K}$ is the unit vector directed along $X$

$$
\hat{K}_{(x y z)}=\left(\begin{array}{l}
K_{x} \\
K_{y} \\
K_{z}
\end{array}\right)=\mathbf{C}_{\gamma} \mathbf{C}_{\beta} \mathbf{C}_{\delta} \mathbf{C}_{\varphi}\left(\begin{array}{l}
0 \\
0 \\
1
\end{array}\right)=\left(\begin{array}{c}
-\sin \gamma \sin \beta \sin \varphi+(\cos \gamma \sin \delta-\sin \gamma \cos \beta \cos \delta) \cos \varphi \\
-\cos \beta \sin \varphi+\sin \beta \cos \delta \cos \varphi \\
\cos \gamma \sin \beta \sin \varphi+(\sin \gamma \sin \delta+\cos \gamma \cos \beta \cos \delta) \cos \varphi
\end{array}\right)
$$

In misaligned shaft system, if friction, inertia and elastic forces of the cross piece were disregarded, the moment acting on the cross piece through driving shaft is equal to the moment acting on driven shaft through the cross piece, and the line of action of the transmitted moment is along the normal unit vector to the cross piece. Therefore, the transmitted moment acting on the driven shaft is

$$
\vec{M}_{(x y z)}=\left(\begin{array}{c}
M_{x} \\
M_{y} \\
M_{z}
\end{array}\right)=\frac{T_{0}-J_{1} \alpha}{\hat{e}_{n(x y z)} \cdot \hat{K}_{(x y z)}} \hat{e}_{n(x y z)}=\frac{T_{0}-J_{1} \alpha}{e_{n x} K_{x}+e_{n y} K_{y}+e_{n z} K_{z}}\left(\begin{array}{c}
e_{n x} \\
e_{n y} \\
e_{n z}
\end{array}\right)
$$

where $M_{x}, M_{y}, M_{z}$ respectively denote the components of the transmitted moment along $x, y, z$. 
When $\delta=0, \varphi \neq 0, \alpha \neq 0, \beta \neq 0$, and $\alpha, \beta$ are small enough so that $\sin \beta \approx \beta, \cos \beta \approx 1$, $\sin \gamma \approx \gamma, \cos \gamma \approx 1$, the components of the transmitted moment with respect to the $x y z$ frame can be obtained using Eq. 5, Eq. 6, Eq. 7, Eq. 9 and Eq. 10, as follows:

$$
\begin{aligned}
& M_{x}=\frac{T_{0}-J_{1} \alpha}{2(\beta \sin \varphi+\cos \varphi)}\left[-\gamma\left(1-\cos 2 \theta_{1}\right)+\gamma \sin ^{2} \varphi\left(1+\cos 2 \theta_{1}\right)-(\beta \cos \varphi-\sin \varphi) \sin 2 \theta_{1}\right] \\
&=\frac{T_{0}-J_{1} \alpha}{2 \cos ^{2} \varphi}\left[-\gamma \cos \varphi\left(1-\cos 2 \theta_{1}\right)+\gamma \sin ^{2} \varphi \cos \varphi\left(1+\cos 2 \theta_{1}\right)-(\beta-\sin \varphi \cos \varphi) \sin 2 \theta_{1}\right] \\
& M_{y}=\frac{T_{0}-J_{1} \alpha}{2(\beta \sin \varphi+\cos \varphi)}\left[\left(\beta \cos ^{2} \varphi-\beta \sin ^{2} \varphi-\sin \varphi \cos \varphi\right)\left(1+\cos 2 \theta_{1}\right)+\gamma \cos \varphi \sin 2 \theta_{1}\right] \\
&=\frac{T_{0}-J_{1} \alpha}{2 \cos ^{2} \varphi}\left[\left(\beta \cos ^{3} \varphi-\sin \varphi \cos ^{2} \varphi\right)\left(1+\cos 2 \theta_{1}\right)+\gamma \cos ^{2} \varphi \sin 2 \theta_{1}\right] \\
& M_{z}= \frac{T_{0}-J_{1} \alpha}{2(\beta \sin \varphi+\cos \varphi)}\left[\left(\cos ^{2} \varphi+2 \beta \sin \varphi \cos \varphi\right)\left(1+\cos 2 \theta_{1}\right)+\left(1-\cos 2 \theta_{1}\right)+2 \gamma \sin \varphi \sin 2 \theta_{1}\right] \\
&= \frac{T_{0}-J_{1} \alpha}{2 \cos ^{2} \varphi}\left[\left(\cos { }^{3} \varphi+\beta \sin \varphi \cos ^{2} \varphi\right)\left(1+\cos 2 \theta_{1}\right)-(\beta \sin \varphi-\cos \varphi)\left(1-\cos 2 \theta_{1}\right)+\right. \\
&\left.\quad 2 \gamma \sin \varphi \cos \varphi \sin 2 \theta_{1}\right]
\end{aligned}
$$

When $\varphi=0, \delta=0$ and $\alpha, \beta$ are small, the components of $\vec{M}$ are given by

$$
\begin{aligned}
M_{x} & =\left(T_{0}-J_{1} \alpha\right)\left(-\gamma \sin ^{2} \theta_{1}-\beta \sin \theta_{1} \cos \theta_{1}\right)=\frac{T_{0}-J_{1} \alpha}{2}\left[-\gamma\left(1-\cos 2 \theta_{1}\right)-\beta \sin 2 \theta_{1}\right] \\
M_{y} & =\left(T_{0}-J_{1} \alpha\right)\left(\gamma \sin \theta_{1} \cos \theta_{1}+\beta \cos ^{2} \theta_{1}\right)=\frac{T_{0}-J_{1} \alpha}{2}\left[\gamma \sin 2 \theta_{1}+\beta\left(1+\cos 2 \theta_{1}\right)\right] \\
M_{z} & =\left(T_{0}-J_{1} \alpha\right)\left[1-\left(\gamma \sin \theta_{1}+\beta \cos \theta_{1}\right)^{2}\right] \\
& =\left(T_{0}-J_{1} \alpha\right)\left[1-\frac{\gamma^{2}}{2}\left(1-\cos 2 \theta_{1}\right)-\frac{\beta^{2}}{2}\left(1+\cos 2 \theta_{1}\right)-\beta \gamma \sin 2 \theta_{1}\right]
\end{aligned}
$$

When $\alpha=0, \beta=0, \delta=0, \varphi \neq 0$, the components of $\vec{M}$ are given by

$$
\begin{aligned}
& M_{x}=\left(T_{0}-J_{1} \alpha\right)\left(\operatorname{tg} \varphi \sin \theta_{1} \cos \theta_{1}\right)=\frac{T_{0}-J_{1} \alpha}{2}\left(\operatorname{tg} \varphi \sin 2 \theta_{1}\right) \\
& M_{y}=\left(T_{0}-J_{1} \alpha\right)\left(-\sin \varphi \cos ^{2} \theta_{1}\right)=\frac{T_{0}-J_{1} \alpha}{2}\left[-\sin \varphi\left(1-\cos 2 \theta_{1}\right)\right] \\
& M_{z}=\left(T_{0}-J_{1} \alpha\right) \frac{1-\sin ^{2} \varphi \cos ^{2} \theta_{1}}{\cos \varphi}=\left(T_{0}-J_{1} \alpha\right) \frac{1-V \cos 2 \theta_{1}}{U}
\end{aligned}
$$

\section{Simulation}

When setting a list of values of $T_{0}, J_{1}, \alpha, \varphi, \beta, \gamma$, Eq. 11, Eq. 12, Eq. 13, can be simulated by MATLAB, and then the fluctuation of transmitted moment ( both lateral and torsional components ) will be see more clearly. Here a numerical illustration which referred to as the "laboratory model" will be given, the parameter values are $T_{0}=0.3 \mathrm{~N} \cdot \mathrm{m}, J_{1}=2.0 \times 10^{-7} \mathrm{~kg} \cdot \mathrm{m}^{2}, \alpha=30 \mathrm{rad} / \mathrm{s}^{2}, \varphi=30^{\circ}$, $\beta=0.3^{\circ}, \gamma=0.3^{\circ}$. Shown in Fig. 2 is the fluctuation of transmitted moment for this laboratory model. From Fig. 2, it is seen that the torsional torque transmitted to the driven shaft $M_{z}$ fluctuates between 0.26 and 0.35 , the lateral moment transmitted to the driven shaft $M_{x}$ fluctuates between 0 and 0.09 , the lateral moment transmitted to the driven shaft $M_{y}$ fluctuates between 0 and 0.15 . It is also seen that when $M_{y}$ and $M_{z}$ at the maximum $M_{x}$ is zero, when $M_{y}$ and $M_{z}$ at the least value $M_{x}$ is also zero; the fluctuation of all components of transmitted moment are periodic with time, the periods are variational, they become shorter with time. 


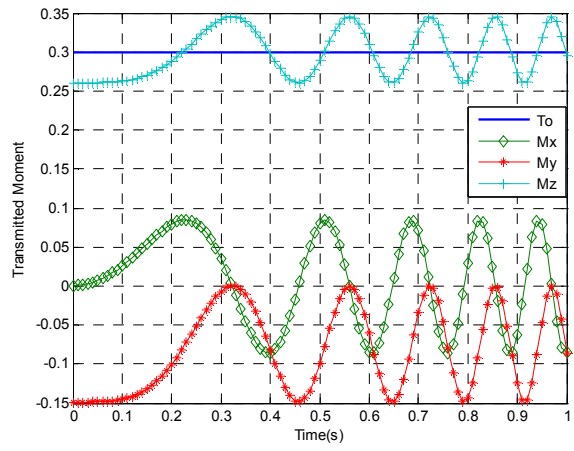

Fig. 2. Fluctuation of transmitted moment

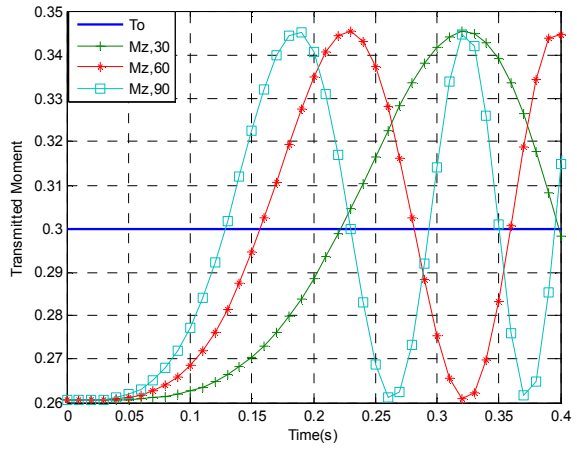

Fig. 3. Plots of the torsional torque

Fig. 3 gives plots of the torsional torque transmitted to the driven shaft with the change of the angular acceleration of the driving shaft. As shown in Fig. 3, the fluctuating amplitudes of transmitted moment do not change, but the fluctuating periods decrease with the increasing of the angular acceleration of the driving shaft.

\section{Conclusions}

This work investigated the moments transmitted by a universal joint in varying operating condition. Both structure misalignment and error misalignment between the two shafts at the universal joint was considered, the expressions of components of the transmitted moment acting on the driven shaft were obtained via method of vector projection. The research work could be offered a foundation to the dynamics problems (torsional vibration, lateral vibration, lateral-torsional coupled vibration and so on) of misaligned shafts driven by universal joint in varying operating condition.

\section{Acknowledgements}

This work was financially supported by Natural Science Foundation of China (Grant No. 50875259) and The Project-sponsored by SRF for ROCS, SEM.

\section{References}

[1] K. M. Al-Hussain, Dynamic stability of two rigid rotors connected by a flexible coupling with angular misalignment, Journal of Sound and Vibration, Vol. 266 (1955), p. 217-234.

[2] I. Porat, Moment transmission by a universal joint, Mechanism and Machine Theory, Vol. 15 (1980), p. 245-254.

[3] T. Iwatsubo and M. Saigo, Transverse Vibration of a Rotor System Driven by a Cardan Joint, Journal of Sound and Vibration, Vol. 95 (1984), p. 9-18.

[4] A. J. Mazzei, A. Argento and R. A. Scott, Dynamic stability of a rotating shaft driven through a universal joint, Journal of Sound and Vibration, Vol. 222 (1999), p. 19-47.

[5] H. Ota and M. Kato, Lateral vibration of a rotating shaft driven by a universal joint - 1st report, generation of even multiple vibrations by secondary moment, Bulletin of JSME, Vol. 27 (1984), p. 2002-2007.

[6] M. Kato and H. Ota, Lateral excitation of a rotating shaft driven by a universal joint with friction, Journal of Vibration and Acoustics, Vol. 112 (1990), p. 298-303.

[7] P. P. Sheu, W. H. Chieng and A. C.Lee, Modeling and Analysis of the Intermediate Shaft between Two Universal Joints, Journal of Vibration and Acoustics, Vol. 118 (1996), p. 88-99. 